\title{
Servicios ecosistémicos con potencial turístico del parque nacional Archipiélago Espíritu Santo, México
}

\section{Resumen}

Elizabeth Olmos-Martínez*

Oscar Alfredo Arizpe-Covarrubias

Reyna María Ibáñez Pérez

Universidad Autónoma de Baja California Sur

Alfredo Ortega-Rubio

Centro de Investigaciones Biológicas del Noroeste, S.C.

La biodiversidad en todos sus niveles aporta beneficios a la sociedad de distintas formas, como el caso del área natural protegida parque nacional Archipiélago Espíritu Santo (PNAES), en Baja California Sur, México. Se realizó una descripción y caracterización, con base en el análisis de información ambiental y socioeconómica, como beneficio recreativo derivado de la riqueza biológica de los ecosistemas. Se elaboraron mapas para identificar las zonas de mayor beneficio turístico y de pesca deportiva. Se identificaron seis servicios ecosistémicos provistos por el pNAEs: refugio de especies, belleza escénica, producción de alimentos, recreación, culturales y recursos genéticos. La principal fauna marina como atractivo para actividades recreacionales en el área son ballenas, delfines y lobos marinos. Se registraron 62 especies de peces en fondos blandos, y 121 en zonas de arrecife, siendo Los Islotes la zona de mayor riqueza de peces arrecifales con 74 especies. Existen prestadores de servicios de pesca deportiva en La Paz que utilizan entre 25 y 30 embarcaciones. Los operadores ecoturísticos promueven los servicios ecosistémicos culturales de recreación, entre los que destacan veleo, surfeo, buceo libre y autónomo, kayakismo, canotaje y fondeo de embarcaciones. En Los Islotes, La Ballena, El Gallo y La Gallina se da la mayor actividad de buceo autónomo y libre. Al PNAEs arriban en promedio 15 embarcaciones al día, las cuales trasportan entre seis y diez turistas. En 2013 el número de visitantes ascendió a 16065 , según los registros de cobro de derechos. El pnAEs retoma cada vez mayor importancia por sus atractivos turísticos y la belleza de sus paisajes naturales.

\section{Palabras clave}

Valoración paisajística, recursos naturales, potencialidad turística.

*Correo electrónico: eolmos@uabcs.mx, oarizpe@uabcs.mx, ribanez@uabcs.mx, aortega@cibnor.mx

Recibido: 30/03/2014 · Aceptado: 27/04/2014




\title{
Ecosystem services with tourism potential of the Espíritu Santo Archipelago natural park in Baja California Sur, México.
}

Elizabeth Olmos-Martínez*

Oscar Alfredo Arizpe-Covarrubias

Reyna María Ibáñez Pérez Universidad Autónoma de Baja California Sur

Alfredo Ortega-Rubio

Centro de Investigaciones Biológicas del Noroeste, S.C.

\begin{abstract}
Biodiversity at all levels brings benefits to society in various ways as can be appreciated in the national park. Based on the analysis of environmental and socio-economic information as well as recreational benefits derived from biologically rich ecosystems, a description and characterization was developed. Maps were drawn to identy the areas with the greatest tourism and fishing potential. Six ecosystem services were identified within the park: species shelter, scenic beauty, food production, recreation, culture and genetic resources. The most important attraction for reacreational activities in terms of marine fauna in the area are whales, dolphins, and sea lions. 62 soft bottom and 121 reef fish species were recorded where the "Los Islotes" area with 74 species represented the largest richness in coral reef fish. Sport fishing service providers of La Paz use between 25 and 30 boats and eco tour operators promote cultural ecosystem recreational services which include sailing, surfing, snorkeling, scuba diving, kayaking, boating and boat anchoring. Snorkeling and scuba diving is most common in the Los Islotes, La Ballena, El Gallo y La Gallina areas. As an average the park receives 15 boats carrying between six and ten tourists each. According to fee collection records, in 2013 the number of visitors amounted to 16,065 which shows that the park has become increasingly more important due to its tourist attractions and the beauty of its natural landscapes.
\end{abstract}

\section{Key words}

Recreational atractions, natural protected area, tourism, sport fishing, biodiversity.

*E-mail: eolmos@uabcs.mx, oarizpe@uabcs.mx, ribanez@uabcs.mx, aortega@cibnor.mx 


\section{Introducción}

La Comisión Nacional de Áreas Naturales Protegidas (Conanp) estima que alrededor de 5.5 millones de turistas visitan anualmente las áreas naturales protegidas (ANP) de México, lo cual deja una derrama económica calculada en tres mil millones de pesos anuales por la prestación directa del servicio turístico. Este segmento del turismo debe verse como una gran oportunidad para el desarrollo del país (Conanp-Semarnat, 2007).

La biodiversidad en todos sus niveles (ecosistemas, especies y genes) aporta beneficios a la sociedad de diferentes formas, por ejemplo a través de productos que se pueden obtener de los ecosistemas, por medio de la prestación de servicios ambientales o por el aprovechamiento consuntivo y no consuntivo de especies, entre muchas otras fuentes de valor, como la valoración económica de los ecosistemas (Costanza et al., 1997).

La parte exclusivamente marina que rodea el Anp llamada complejo insular Espíritu Santo (CIES) ha sido recientemente declarada área federal protegida con la modalidad de parque nacional Archipiélago Espíritu Santo (PNAES), dada la importancia terrestre y marina de la zona. Los principales procesos socioeconómicos internos que tienen lugar en el CIES y el PNAEs son los derivados de los sectores primario y terciario de la economía, en especial la pesca artesanal y la prestación de servicios turísticos orientados a la naturaleza.

\section{Área de estudio}

El pnaEs está ubicado en el Golfo de California, frente a las costas del estado de Baja California Sur (B.C.S.), México. El área de influencia del complejo comprende el municipio de La Paz, de cuya ciudad está separada por solo $25 \mathrm{~km}$ en línea recta. Esta área se localiza entre los $24^{\circ} 24^{\prime}$ y los $24^{\circ} 36^{\prime}$ de latitud norte y los $110^{\circ} 18^{\prime}$ y los $110^{\circ} 27^{\prime}$ de longitud oeste, separada de la península de Pichilingüe por el canal de San Lorenzo, de aproximadamente $8 \mathrm{~km}$ de ancho, y constituye parte de los límites orientales de la bahía de La Paz (Conanp-Semarnat, 2006) (figura 1). 
Complejo Insular Espíritu Santo

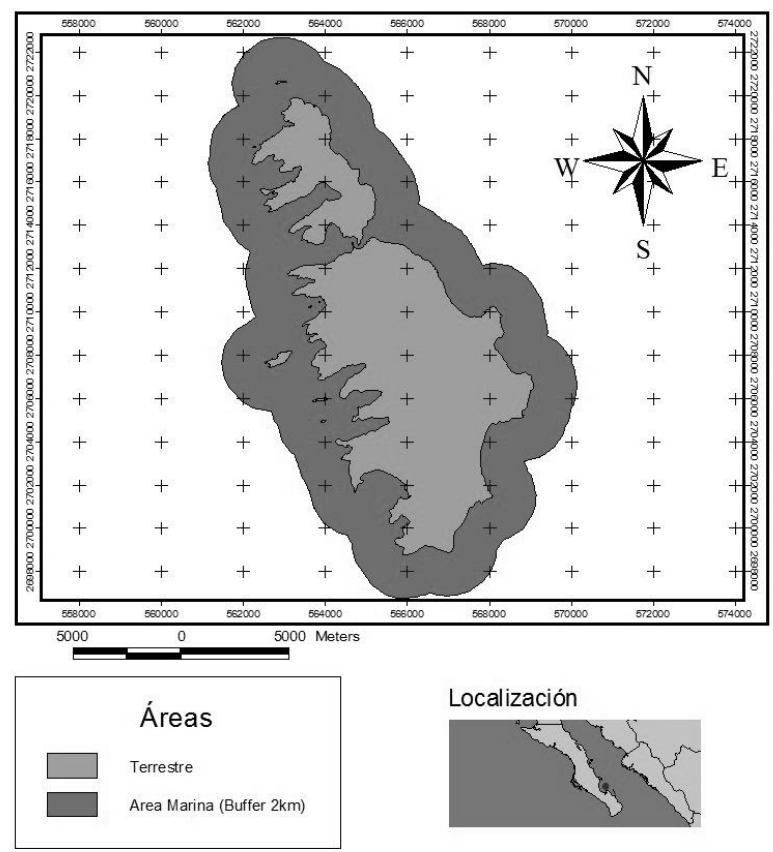

Fuente: Imagen Satelital SPOT del 16 de abril 2008 Escala 1:20,000

Sistema de Coordenadas UTM

Datum WGS84

Elaboró: FMCV

Fuente: Cervantes (2011).

Figura 1. Localización geográfica del cies en donde se especifica el área terrestre y el área de influencia marina (PNAES)

Destaca la importancia del área marina del CIES, es decir, el PNAEs, por lo que se consideró una línea de $2 \mathrm{~km}$ de distancia de la línea de costa de la isla. Esta medida se tomó a partir de la distancia resultante de la punta de La Ballena, que es el mayor de los islotes del complejo, a la línea de costa de la isla Espíritu Santo. Por lo tanto, el área de estudio contempla también parte del PNAEs declarado como ANP en 2007, donde se tiene en cuenta exclusivamente la zona marina del CIEs (Presidencia de la República, 2007). 


\section{Metodología}

La descripción y caracterización se funda en la recopilación, integración y análisis de información ambiental, basada en los recursos naturales marinos como atractivo turístico y beneficio recreativo, además de información económica y social para conocer el provecho socioeconómico de las actividades turísticas y de pesca deportiva derivadas de la riqueza biológica de los ecosistemas. Se realizó un mapa del pNAEs para identificar las zonas de mayor atractivo turístico, en particular en las actividades turísticas y de pesca deportiva, por medio de material cartográfico a través de análisis, interpretación y digitalización de imágenes satelitales spot proporcionado por la Conanp y de información brindada por la Sociedad de Historia Natural Niparajá, A.C. El mapa ayudó en el examen de zonas prioritarias para la conservación presumiblemente vulnerables por la actividad antropogénica.

Se aplicaron 60 encuestas a los prestadores de servicios turísticos, de un total de 70, con la finalidad de obtener datos cualitativos y cuantitativos (zonas donde realiza actividades turísticas, zonas más visitadas, promedio mensual de turistas, lugar de procedencia del turista, actividad mayormente atractiva, tipo de embarcación, meses de mayor afluencia, etc.) para caracterizar y describir los atractivos turísticos y de pesca deportiva que dependen directamente del ecosistema del PNAES y que brindan beneficios recreativos y económicos a partir de los servicios ecosistémicos del área para la ciudad de La Paz, B.C.S.

\section{Resultados}

\section{Análisis descriptivo de la biodiversidad como atractivo recreacional}

Se identifican seis servicios ecosistémicos provistos por el pNAEs: 1) refugio de especies, 2) belleza escénica, 3) producción de alimentos, 4) recreación, 5) culturales y 6) recursos genéticos, cada uno vinculado a funciones ecológicas y a usuarios específicos (Hernández-Trejo, Avilés-Polanco y Almendárez-Hernández, 2012). De acuerdo con Forest Trends y The Katoomba Group (2010), Van den Hove (2009), Keller et al. (2008), Beaumont et al. (2007), Conanp-Semarnat 
(2006) y Daily et al. (1997), las funciones ecológicas y usuarios para cada servicio ecosistémico que aportan las ANP marinas son:

Refugio de especies. Función: hábitat para poblaciones residentes y migrantes. Usuarios/actividad: pesca artesanal, pesca deportiva, ecoturismo y acuacultura. Belleza escénica. Función: oportunidades para actividades recreativas. Usuarios/actividad: senderismo y campismo.

Producción de alimentos. Función: producción de materia prima extraíble. Usuarios/actividad: pesca artesanal, pesca deportiva y acuacultura.

Recreación. Función: oportunidad para actividades recreativas. Usuarios/actividad: turismo alternativo y pesca deportiva.

Culturales. Función: actividades para usos no comerciales. Usuarios/actividad: ecoturismo e investigación.

Recursos genéticos. Función: fuentes de material biológico y productos únicos. Usuarios/actividad: investigación y bioprospección.

En B.C.S., las características de la bahía de La Paz permiten la presencia, ya sea temporal o permanente, de diversos mamíferos marinos; algunas especies encontradas en la zona son consideradas comunes, mientras que otras escasas o raras, dependiendo de la frecuencia con la que son observadas (Urbán et al., 1994). En el área de Los Islotes se halla establecida una población de lobos marinos de California (Zalophus californianus) y dos o tres ejemplares de elefantes marinos (Arizpe, 1998). Esta colonia de lobos marinos reviste especial interés por ser la lobera reproductora más sureña de toda el área de distribución de la subespecie, además de una de las colonias reproductoras más pequeñas de México (Aurioles, 1994).

Se han reportado 26 especies de cetáceos para la bahía de La Paz. Algunas de ellas se encuentran solo durante la temporada fría por ser especies migrantes, como la ballena gris (Eschrichtius robustus), la ballena azul (Balaenoptera musculus) y la ballena jorobada (Megaptera novaeangliae). Las observadas con mayor frecuencia durante las temporadas fría y cálida son el rorcual tropical (Balaenoptera edeni), el delfín común de rostro largo (Delphinus capensis) y el tursión costero y oceánico (Tursiops truncatus). La orca (Orcinus orca) es otra 
especie que se presenta de manera esporádica en la bahía, sobre todo cerca de Los Islotes (Conanp-Semarnat, 2011).

Los peces registrados en el PNAES, específicamente en fondos blandos, son 62 especies, mientras que en zonas de arrecife hay 121 especies, donde Los Islotes constituye la zona de mayor riqueza de peces arrecifales con 74 especies y las familias mejor representadas son Serranidae, Labridae, Pomacentridae, Lutjanidae, Haemulidae y Scaridae (Conanp-Semarnat, 2011).

Los corales rígidos y blandos configuran uno de los mayores atractivos turísticos del PNAES, siendo el género Pocillopora el más abundante en el Golfo de California que, aunque no conforma arrecifes, se presenta en formaciones más o menos masivas en la Bahía San Gabriel, Roca Swany, Las Navajas y El Coralito (Conanp-Semarnat, 2000). Reyes et al. (2007) reportan nueve especies correspondientes a cinco géneros dentro de la zona del PNAEs, entre las cuales se hallan cinco especies hermatípicas: Pocillopora captiata, P. damicornis, P. meandrina, P. verrucosa y Porites panamensis.

Estas formaciones coralinas son de gran relevancia ya que son utilizadas como hábitat, sitios de reproducción, alimentación y refugio para muchas otras especies como camarones y moluscos (Conanp-Semarnat, 2011).

Para la zona del PNAES se han reportado en total 64 especies de equinodermos, 20 especies de la clase Asteroidea, 13 de la Ophuiuroidea, 10 de la Echinoidea y 21 de la Holoturoidea, dentro de las que destacan dos especies endémicas: el asteroideo (Mitrodia enriquecasoi) y el holoturoideo (Laetmogone scotoeides) (Conanp-Semarnat, 2011).

En el PNAES se han registrado importantes especies consideradas bajo alguna categoría de protección según la norma oficial mexicana Nom-059-Semarnat-2010, como son la madreperla (Pinctada mazatlanica), el pepino de mar (Istichopusfuscus), peces como la damisela azul y amarilla (Chromis Iimbaughi), el lobo marino de California (Zalophus californianus), el ángel rey (Holacanthus passer) y el ángel cortés (Pomacanthus zonipectus); las tortugas caguama (Caretta caretta), prieta (Chelonia agassizi), laúd (Dermochelys coriácea), carey (Eretmochelys imbrincata) y golfina (Lepidochelys olivácea), además del murciélago pescador (Myotis vivesi), están catalogadas en peligro de extinción; dentro de las aves, los petreles de tormenta (O. melania y O. microsoma) 164 se encuentran en la categoría de amenazadas, y las gaviotas ( $L$. livens y $L$. 
hermanni), la garza (E. rufescens) y el rascón picudo ( $R$. longirostris), en la de protección especial (Conanp-Semarnat, 2011).

El clima es cálido y seco, con lluvias escasas. La temporada de lluvias va de junio a octubre, asociada a los vientos del sur y la incidencia de fenómenos de baja presión; la precipitación media anual es de $180 \mathrm{~mm}$ y la evaporación media anual de $300 \mathrm{~mm}$. La temperatura máxima ambiental promedio de $30{ }^{\circ} \mathrm{C}$ se reporta al final del verano y la mínima de $17^{\circ} \mathrm{C}$ al comienzo del invierno (Conanp-Semarnat, 2000). La temperatura superficial del mar en invierno varía de 19 a $21{ }^{\circ} \mathrm{C}$, durante la primavera de 21 a $24{ }^{\circ} \mathrm{C}$, en verano de 24 a $28{ }^{\circ} \mathrm{C}$, mientras que en otoño inicia el descenso de la temperatura (Conanp-Semarnat, 2011).

\section{Análisis descriptivo de las actividades recreativas de pesca deportiva y turismo}

\section{Pesca deportiva}

Se tiene registro de la existencia de varios prestadores de servicios de pesca deportiva en La Paz que utilizan entre 25 y 30 embarcaciones, los cuales están organizados en más de cuatro cooperativas y dos empresas. Las especies de interés para esta actividad son: marlin azul (Makaria nigricans), marlin rayado (Tretapturus audax), marlin negro (Makaria indica), pez vela (Istiophorus platypterus), pez espada (Xiphias gladitus) y pez dorado (Coryphaena hippurus) (Conanp-Semarnat, 2011); esta práctica se concentra de febrero a octubre (Conanp-Semarnat, 2011; Sociedad de Historia Natural Niparajá, 2005).

La pesca deportiva, a diferencia de la pesca ribereña, ha sido menos estudiada y caracterizada, según estudios realizados por el Centro Interdisciplinario de Ciencias Marinas, del Instituto Politécnico Nacional, y publicados en el programa de manejo del cies (Conanp-Semarnat, 2000). Se ha evidenciado la relevancia del área para esta actividad que es considerada una de las más importantes en la bahía.

De acuerdo con las encuestas aplicadas a los prestadores de servicios en las islas del complejo, la relación que estos mantienen con los pescadores que operan en las islas es buena, basada en el respeto y la independencia, tanto en sus respectivas actividades como en los sitios donde acampan. A su vez, los pescadores artesanales encuestados manifiestan la competencia desleal que 
ejerce la pesca deportiva mediante el buceo libre, por lo selectiva que resulta, así como por la frecuencia con que se realiza, en ciertas épocas del año por numerosos grupos de deportistas, lo que disminuye la posibilidad de éxito de la pesca artesanal con línea.

\section{TURISMO}

Por su parte, la Conanp (2011) ha señalado un notable aumento de las actividades turístico-recreativas en ANP. Tanto el incremento de visitas, como el interés por manejarlo apropiadamente, es un fenómeno de gran importancia en México. Estudios de la Secretaría de Turismo han encontrado que las anp son los destinos favoritos para llevar a cabo actividades de ecoturismo y turismo de aventura (Semarnat/Conanp, 2007), por lo que estas se han visto como una oportunidad más para el desarrollo del país.

Existe una estrecha relación entre la protección del patrimonio natural del área de Espíritu Santo y las actividades turísticas en el sitio. El ciEs es visitado por un número creciente de turistas que realizan diversas actividades orientadas a la observación y disfrute de la naturaleza, la mayoría de las cuales tienen lugar en la zona marina y están basadas en el aprovechamiento recreativo de la belleza escénica de la costa y playas, en la biodiversidad y variedad de hábitats marinos, en los avistamientos de cetáceos y en la presencia de la colonia de lobos marinos en Los Islotes. Los visitantes llegan al CIES y al PNAEs atraídos por estos valores naturales y por la oportunidad de disfrutar de ellos en una condición de mínima interferencia humana (Sociedad de Historia Natural Niparajá, 2005).

El PNAES es considerado un sitio ideal para desarrollar actividades recreativas, y uno de los principales atractivos naturales de La Paz. Los operadores ecoturísticos promueven los servicios ecosistémicos culturales de recreación que proporciona el sitio mediante la comercialización de actividades acuáticas para disfrutar su biodiversidad, entre las que destacan el veleo, el surfeo, el buceo libre y el autónomo, el kayakismo, el canotaje y el fondeo de embarcaciones (Conanp-Semarnat, 2000, 2006 y 2011).

Este tipo de turismo, basado en el aprovechamiento de varios de los servicios ambientales del área, ha experimentado un incremento significativo en la 166 última década. Según las encuestas, las actividades de veleo, buceo, kayakismo, 
campismo y cruceros naturalistas que se llevan a cabo en el PNAEs generan una derrama económica importante para la ciudad de La Paz, donde se encuentran los centros de operación de la mayoría de las empresas que ofrecen los servicios. Para ello, acceden a las islas en yates y embarcaciones menores, y concentran sus actividades en pocos sitios del complejo insular. Las zonas donde existe mayor actividad de buceo autónomo y libre son Los Islotes, La Ballena, El Gallo y La Gallina. Los lugares más utilizados para el anclaje de embarcaciones son Ensenada Grande, El Cardonal, La Partida y El Candelero (figura 2).

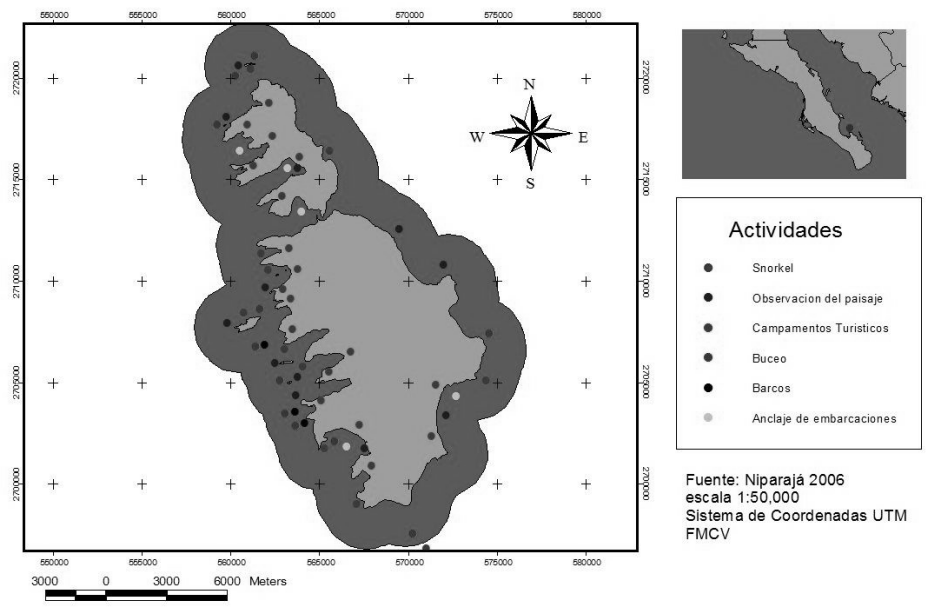

Fuente: Cervantes (2011).

Figura 2. Zonas de principales actividades turísticas en el PNAES

El aprovechamiento turístico de la colonia de lobos marinos de Los Islotes brinda un ejemplo de la relevancia económica del área. A esta colonia reproductiva -una de las más visitadas en el golfo- arriban en promedio 15 embarcaciones al día, las cuales trasportan entre seis y diez turistas (Conanp-Semarnat, 2006 y 2011). Se estima que la derrama económica de este sitio es de entre 1000 y 1500 dólares diarios durante los meses de verano (Conanp-Semarnat, 2011). 
Para el periodo 2009-2010, la dirección administrativa del PNAES registró un total de 54 empresas turísticas y otorgó 265 autorizaciones a empresas prestadoras de servicios turísticos. La mayoría de las organizaciones realiza sus actividades durante todo el año y algunas solo de octubre a mayo (ConanpSemarnat, 2011). De acuerdo con datos de la Dirección del Área de Protección de Flora y Fauna Islas del Golfo de California, B.C.S. (APFFIGC-BCS) y el PNAEs, el número de visitantes para el año 2013 ascendió a 16065 personas, con base en los registros de cobro de derechos, el cual generó una recaudación por la cantidad de 772096 pesos. Sobre la nacionalidad de los turistas, la dirección del ANP nos comenta que a la fecha no tiene registro alguno que pueda diferenciar entre nacionales o extranjeros.

Según el cuestionario aplicado a los prestadores de servicios, el turismo procedente de Estados Unidos es el de mayor afluencia a la isla; los motivos centrales de la visita de los turistas son la gran diversidad biológica de la zona y las condiciones favorables para el desarrollo de actividades subacuáticas; mientras que las principales actividades realizadas son la visita a la lobera en Los Islotes y la práctica de snorkel, buceo autónomo y buceo libre.

Existe una cantidad considerable de embarcaciones particulares que llegan al PNAES a lo largo del año, particularmente veleros y yates. Este tipo de visitantes permanece en las ensenadas y bahías más protegidas del cIEs.

\section{Conclusión y discusión}

Los instrumentos de intervención más utilizados para ofertar espacios de uso recreativo son la construcción y conservación de zonas verdes urbanas y la declaración y mantenimiento de espacios naturales protegidos. El CIES y el PNAES, al ser un área natural protegida con doble decreto de protección -por un lado el CIES se incluye en las islas del APFFIGC-BCS y más recientemente el decreto de PNAES-, constituye una zona destinada a la salvaguarda de los ecosistemas que, por su representatividad biogeográfica nacional, reúnen condiciones de especial valor natural y favorecen la realización de actividades científicas, educativas y de recreo compatibles con la protección y la preservación de sus recursos naturales; a su vez, es una zona donde se pueden encontrar especies endémicas del estado y una gran biodiversidad en su contiguo marino (Conanp-Semarnat, 2011). 
El PNAES cada vez retoma mayor importancia por sus atractivos turísticos y por la belleza de sus paisajes naturales tanto terrestres como marinos. Su cercanía a la ciudad de La Paz se traduce en facilidad de acceso para el turismo nacional e internacional.

En el PNAES se distribuyen especies consideradas bajo alguna categoría de protección. Las áreas de más afluencia y con mayor presión por parte de los sectores en el ciEs y su área adyacente marina son las más cercanas a la línea de costa y la zona federal marítimo-terrestre, principalmente con usuarios del sector pesquero y turístico. Una de las actividades con mayor presencia en el PNAES es el turismo debido a que está en crecimiento dentro del ANP, a pesar de que esta actividad se enfoca al ecoturismo o turismo de aventura. La demanda de las actividades depende directamente de la calidad del entorno natural que brinda el ecosistema. La relación entre los atributos naturales del sitio y el mantenimiento de las actividades productivas puede ser expresada en términos del concepto servicios de los ecosistemas.

En 2009, dada la importancia del Anp, Hernández-Trejo, Avilés-Polanco y Almendárez-Hernández (2012) efectuaron un estudio sobre la valoración económica del PNAEs utilizando el método costo de viaje. Es conocido que la valoración económica es una herramienta que ayuda a diseñar instrumentos económicos de política ambiental orientados a modificar patrones de consumo. Del mismo modo favorece la estimación del monto económico de posibles daños sobre los servicios ecosistémicos (Seenprachawong, 2003). Como propuesta de la economía ambiental existen métodos de valoración económica en la búsqueda de instrumentos que coadyuven a la conservación a partir del valor económico total, ya sea de uso directo, indirecto, de herencia, opción, existencia y uso pasivo (Heal et al., 2005; Costanza et al., 1997); asimismo, estimar el valor económico asociado a un servicio ecosistémico puede realizarse mediante dos enfoques metodológicos (de mercado o de no mercado) propuestos por la economía ambiental (Turner, Pearce y Bateman, 1994).

Los resultados obtenidos por Hernández-Trejo, Avilés-Polanco y Almendárez-Hernández (2012), dentro del análisis descriptivo de las encuestas, indican que $62 \%$ de los turistas visita el ANP por primera vez y $67 \%$ considera que el PNAES es muy importante en cuanto a biodiversidad; $82 \%$ de los visitantes es extranjero, $14 \%$ corresponde a turismo nacional con una edad promedio de 43 
años, $62 \%$ de los visitantes es del género masculino y $38 \%$ del femenino, $52 \%$ tiene un grado de escolaridad alto ubicado con nivel superior y $30 \%$ con nivel posgrado. El mismo estudio resalta en sus conclusiones que el valor crematístico calculado a través de actividades ecoturísticas para la biodiversidad acuática que resguarda el PNAES reconoce que estas actividades generan beneficios para los visitantes y para los individuos locales que disfrutan de la proveeduría de servicios recreativos culturales. Este hecho exterioriza la importancia y función que desempeña el PNAES en materia de conservación de ecosistemas y hábitats para la que fue concebido. Los valores económicos recreativos agregados por tipo de turista difieren debido a la proporción de visitantes nacionales y extranjeros.

Los mismos autores del estudio comentan que las cuotas actuales coadyuvan al desarrollo de actividades de conservación y educación ambiental; de incrementarse, también lo haría el monto recaudado y se abriría la posibilidad de ampliar el gradiente de acciones de manejo y conservación dentro del PNAEs. El cobro diferenciado en las cuotas de acceso es posible debido al carácter inelástico de las funciones de demanda y los montos de la disposición a pagar para turistas extranjeros y nacionales. Los aumentos representan incremento en la recaudación, lo que permitirá implementar tres posibles estrategias. La primera, un aumento en las cuotas de acceso por tipo de actividad que se realice; la segunda, la posibilidad de proponer cobros diferenciados por tipo de turista (local, nacional o extranjero); por último, se pueden establecer precios diferenciados en las cuotas según la temporada del año: cuotas bajas en temporada baja y cuotas más altas en temporada estival. La decisión de modificar las cuotas de acceso dependerá del marco temporal, social, político, económico y ambiental que impere en el momento; teniendo en cuenta que el beneficio a largo plazo es la conservación de las especies para que las actividades acuáticas turísticas en el PNAEs se mantengan.

Dado lo anterior, el estudio de valoración económica referenciado muestra el valor económico, los beneficios recreativos, sociales, culturales y ambientales del CIES y el PNAEs en función de las respuestas de los propios visitantes, lo que sin duda merece atención de parte de las autoridades e intensifica la importancia y justificación de los decretos para protección de las zonas terrestre y 170 marítima del área de estudio a partir de la riqueza del ecosistema. 
La Conanp (2006) menciona que el PNAEs es considerado uno de los sitios más biodiversos y productivos del Golfo de California, ya que en él se encuentran ecosistemas únicos y hábitats vulnerables. Dentro de su polígono hay un número significativo de especies, incluyendo endémicas, amenazadas y en peligro de extinción; así como otras de valor comercial, cultural y recreativo. Lo anterior define al PNAEs como área marina concentradora de distintas especies (alrededor de 1 589, entre ellas algas, esponjas, moluscos, crustáceos, anfibios, reptiles terrestres y marinos, aves terrestres y marinas, mamíferos terrestres y marinos, cindarios, fanerógamas, anélidos, equinodermos y peces, algunas protegidas por normas oficiales mexicanas).

Con base en los resultados del análisis, se pueden efectuar las siguientes recomendaciones: 1) aplicar a la pesca deportiva la misma restricción de la pesca artesanal vigente en el área marina protegida y 2) asignar tres embarcaciones de vigilancia tanto para supervisar que se cumpla la restricción de pesca en el área marina protegida como para evitar la sustracción de especies de flora y fauna marina por parte de buzos y esnorquelistas, las cuales complementarían a las tres embarcaciones ya existentes, para vigilancia y otras actividades, en la administración del PNAEs, logrando mayor eficiencia en estas tareas.

\section{Fuentes consultadas}

Arizpe Covarrubias, O. (1998). "El ambiente marino”, en Diagnóstico ambiental de Baja California Sur. La Paz, B.C.S.: Talleres Gráficos de la Universidad Autónoma de Baja California Sur (UABCS), 245-301.

Aurioles Gamboa, D. (1994). "Biología y ecología del lobo marino de California Zalophus californianus en la Bahía de La Paz, B.C.S, México”, en Coloquio sobre la Bahía de La Paz. Programas y resúmenes. La Paz, B.C.S.: uABCs/Centro Interdisciplinario de Ciencias Marinas (Cicimar)-Instituto Politécnico Nacional (IPN), 35-41.

Beaumont, N.J. et al. (2007). "Identification, Definition and Quantification of Goods and Services Provided by Marine Biodiversity: Implications for the ecosystem approach en EE.UU.”. Marine Pollution Bulletin, 54, 253265. 
Cervantes Villegas, F. (2011). Caracterización y diagnóstico ambiental del complejo insular Espíritu Santo, municipio de La Paz B.C.S, México. La Paz, B.C.S.: UABCS.

Conanp-Semarnat (2000). Programa de Manejo Complejo Insular del Espíritu Santo. México: Comisión Nacional de Áreas Naturales Protegidas-Secretaría de Medio Ambiente y Recursos Naturales.

(2006). Estudio Previo Justificativo para el Establecimiento del Área Natural Protegida "Parque Nacional Espíritu Santo". México: Comisión Nacional de Áreas Naturales Protegidas-Secretaría de Medio Ambiente y Recursos Naturales.

------- (2007). Programa de Turismo en Áreas Protegidas 2006-2012. México: Comisión Nacional de Áreas Naturales Protegidas-Secretaría de Medio Ambiente y Recursos Naturales.

(2011). Programa de Manejo del Parque Nacional exclusivamente la Zona Marina del Archipiélago de Espíritu Santo. México: Comisión Nacional de Áreas Naturales Protegidas-Secretaría de Medio Ambiente y Recursos Naturales.

Costanza, R. et al. (1997). “The Value of the World's Ecosystem Services and Natural Capital”. Nature, 387, 253-260.

Daily, G.C. et al. (1997). "Ecosystem Services: Benefits Supplied to Human Societies by Natural Ecosystems". Issues in Ecology, 2, 18-25.

Forest Trends y The Katoomba Group (2010). Payments for Ecosystem Services: Getting Started in Marine and Coastal Ecosystems. A Primer. Washington, D.C.: The Katoomba Group/Marine Ecosystem Services Program/ Forest Trends.

Heal, G.M. et al. (2005). Valuing Ecosystem Services: Toward Better Environmental Decision-Making. Washington, D.C.: The National Academies Press.

Hernández-Trejo, V., G. Avilés-Polanco y M.A. Almendárez-Hernández (2012). "Beneficios económicos de los servicios recreativos provistos por la biodiversidad acuática del parque nacional Archipiélago Espíritu Santo". Estudios Sociales, XX (40), 155-177.

Keller, B.D. et al. (2008). "Marine Protected Areas", en S. Herrod y J.M. West (eds.). Preliminary Review of Adaptation Options for Climate-Sensitive Ecosystems and Resources. Washington, D.C.: U.S. Climate Change 
Science Program/The Subcommittee on Global Change Research, 8-18-95.

Presidencia de la República (2007). "DECRETO por el que se declara área natural protegida, con la categoría de Parque Nacional exclusivamente la zona marina del Archipiélago de Espíritu Santo, ubicado en el Golfo de California, frente a las costas del Municipio de La Paz, Baja California Sur”. Diario Oficial de la Federación, DCXLIV (7), jueves 10 de mayo, 6-12.

Reyes Bonilla, H. et al. (2007). "Línea base de información biofísica de Espíritu Santo", en Pescando información en Espíritu Santo: Generación de información socioeconómica de gobernabilidad y biofísica. La Paz, B.C.S.: Sociedad de Historia Natural Niparajá, 10-26.

Secretaría del medio ambiente y recursos naturales (SEMARNAT) / Comisión nacional de áreas naturales protegidas (CONANP). (2007). Programa de turismo en áreas protegidas 2006-2012. 18 pp.

Seenprachawong, U. (2003). "Economic Valuation of Coral Reefs at Phi Phi Islands, Thailand”. International Journal of Global Environmental Issue, 3, 104-114.

Sociedad de Historia Natural Niparajá (2005). Estudio previo justificativo Parque nacional Espíritu Santo. La Paz, B.C.S.: Sociedad de Historia Natural Niparajá.

Turner, R.K., D. Pearce e I. Bateman (1994). Environmental Economics. An Elementary Introduction. Brighton: Harvester Wheatsheaf.

Urbán Ramírez, J. et al. (1994). Coloquio sobre la Bahía de La Paz. Programas y resúmenes. La Paz, B.C.S.: UABCs/Cicimar-IPN.

Van den Hove, S. (2009). "Deep-Sea Ecosystem Goods and Services: A Challenge to Socio-economics”, en The Value of Our Marine Environment Conference. Estocolmo: Swedish Environmental Protection Agency, 15-28. 